INPLASY

PROTOCOL

To cite: Zeng et al. The optimal oral biopsy site in the diagnosis of oral mucosal autoimmune bullous disorders: a systematic review and metaanalysis. Inplasy protocol 202080024. doi: 10.37766/inplasy2020.8.0024

Received: 07 August 2020

Published: 07 August 2020

Corresponding author: Hongmei Zhou

acomnet@126.com

Author Affiliation:

West China Hospital of Stomatology, Sichuan University, Chengdu, Sichuan, P.R. China

Support: NNSF of China: 81772898

Review Stage at time of this submission: The review has not yet started.

Conflicts of interest:

No conflict of interest in authors.

\section{The optimal oral biopsy site in the diagnosis of oral mucosal autoimmune bullous disorders: a systematic review and meta-analysis}

Zeng, Q1; Liu, J2; Wu, F3; Zhou, $\mathrm{H}^{4}$.

Review question / Objective: Which is the optimal oral biopsy site in the diagnosis of oral autoimmune bullous disorders, perilesional or non-perilesional mucosa?

Condition being studied: Carey B et al. have shown that in the patients with pure gingival mucous membrane pemphigoid, DIF was negative in four of five biopsied specimens from normal buccal tissue while perilesional reflected alveolar mucosa displayed with the highest rate of positive results, indicating that how to select the biopsied site determine the accuracy of diagnostic outcomes. And many researchers have explored the optimal biopsy site and have controversies. However, there is no systematic review of this question.

INPLASY registration number: This protocol was registered with the International Platform of Registered Systematic Review and Meta-Analysis Protocols (INPLASY) on 07 August 2020 and was last updated on 07 August 2020 (registration number INPLASY202080024).

\section{INTRODUCTION}

Review question / Objective: Which is the optimal oral biopsy site in the diagnosis of oral autoimmune bullous disorders, perilesional or non-perilesional mucosa?
Rationale: Oral mucosal autoimmune bullous disorders characterized clinically by blisters and erosions in oral mucosa, and direct immunofluorescence (DIF) still remains the gold diagnosis standard. However, how to select the biopsied site is 
still a question. This systematic review aimed to determine the optimal oral biopsy site in diagnosis for autoimmune bullous diseases and pave the way to help clinicians choose the surgical site.

Condition being studied: Carey B et al. have shown that in the patients with pure gingival mucous membrane pemphigoid, DIF was negative in four of five biopsied specimens from normal buccal tissue while perilesional reflected alveolar mucosa displayed with the highest rate of positive results, indicating that how to select the biopsied site determine the accuracy of diagnostic outcomes. And many researchers have explored the optimal biopsy site and have controversies. However, there is no systematic review of this question.

\section{METHODS}

Search strategy: Pubmed, Embase, Web of Science, and Cochrane Library databases were searched up to 15 October 2020. Take Pubmed as an example, the searching strategy is: \#1 (bullous OR blistering OR vesiculobullous OR vesicular) AND (disease* OR lesion* OR disorder* OR dermatos*) \#2 (pemphigus vulgaris) OR (mucous membrane pemphigoid) OR (cicatricial pemphigoid) \#3 \#1 OR \#2 \#4 immunofluorescence AND (oral OR mouth OR mucous) AND biopsy \#5 (("Sensitivity and specificity"[MeSH] OR predict*[tw] OR diagnos*[tw] OR accura*[tw]) NOT (case report*[Title/Abstract]) \#6 \#3 AND \#4 AND \#5.

Participant or population: Biopsy-proven patients with oral mucosal autoimmune bullous disorders.

Intervention: Undergone oral biopsy from perilesional site.

Comparator: Undergone oral biopsy from non-perilesional site.

Study designs to be included: Observational studies
Eligibility criteria: The literature search strategy was based on the following inclusion criteria: (1) articles enrolling patients undergoing oral biopsy; (2) studies published in English; (3) articles reporting sample adequacy or diagnostic accuracy. Small case series, case report, review articles, and animal models were excluded.

Information sources: Pubmed, Embase, Web of Science, and Cochrane Library databases were searched up to 15 October 2020. References of included studies, relevant reviews and meta-analyses were examined for potential suitable studies. Authors of included studies were contacted to obtain the full text or further information when needed.

Main outcome(s): Diagnostic yield, which was defined as the number of cases in which a DIF was positive divided by the total of number of cases confirmed with oral mucosal autoimmune bullous disorders.

Additional outcome(s): Odds ratios (OR), which was defined as the diagnostic yield of the perilesional site divided by the diagnostic yield of the non-lesional site.

Data management: Screening and extraction of articles were performed by two independent reviewers (Q.X Z, J.J L)who initially read the titles and abstracts of retrieved records for eligibility. When it was unavailable to obtain sufficient information for judgment or data collection from the abstract, full texts of selected studies were retrieved. Reviewers crosschecked their results after selection, and any controversy was resolved through their discussion or consulting a third reviewer or methodologist. If additional or missing information was required in the original study, the corresponding authors were contacted. Collected data items included: study setting (name of first author, publication year, country), design, characteristics of the enrolled patients (number, age, sex ration), lesion data (location, biopsy site), results (DIF positive rate, DIF negative rate), quality of evidence. 
Quality assessment / Risk of bias analysis: The quality of the cohort studies and casecontrol studies was assessed using the Newcastle-Ottawa Scale (NOS). The final performance on the scale was indicated by total score of " *," with studies with a score of 6 or more " * " considered high quality. The quality of the cross-section studies was assessed by the Agency for Healthcare Research and Quality (AHRQ) scale, which contains 11 items. An item was scored " 0 " if it was answered with "NO" or "UNCLEAR" and " 1 " if it was answered with "YES." Article quality was assessed as follows: low quality $=0-3$, moderate quality $=4-7$, and high quality $=$ 8-11.

Strategy of data synthesis: The STATA software (version 15.1, StataCorp, TX, USA) was applied for synthesizing the results of outcomes. For diagnostic yield, $p$-value with $95 \%$ confidence intervals $(\mathrm{Cl})$ was calculated by command of "metaprop". For odds ratio (OR), the meta-analysis was conducted by generic inverse variance method. The results of the pooled effect size were considered to be statistically significant only at $P<0.05$. The random effects model was used and heterogeneity was calculated by 12 test. Heterogeneity was divided into four grades according to the value of 12: no heterogeneity (less than $25 \%)$, low heterogeneity (25\%-49\%), moderate heterogeneity (50\%-74\%) and high heterogeneity ( $75 \%$ or greater). Funnel plot and Egger' test was used to assess the risk of publication bias.

Subgroup analysis: Subgroup analysis was carried out according to different diseases.

Sensibility analysis: Trim and fill method or fail-safe numbers method was considered to have sensibility analysis.

Language: English.

Country(ies) involved: China.

Other relevant information: The GRADE system was employed to assess the evidence quality for prespecified outcomes. And the "Summary of findings" table was provided by GRADEpro Guideline Development Tool (GDT) online software (https://gradepro.org) to summarize the results.

Keywords: biopsy; diagnosis; immunofluorescence ; autoimmune bullous disorders.

Dissemination plans: Not yet.

Contributions of each author:

Author 1 - Qingxiang Zeng - Author 1 searched studies, screened data and drafted the manuscript.

Author 2 - Junjiang Liu - Author 2 searched studies, screened data, and summarized data.

Author 3 - Fanglong Wu - Author 3 provided the structural expertise and review the manuscript.

Author 4 - Hongmei Zhou - The author read, provided feedback and approved the final manuscript. 